\title{
Volume holographic lens spectrum- splitting photovoltaic system for high energy yield with direct and diffuse solar illumination
}

Benjamin D. Chrysler, Yuechen Wu, Zhengshan Yu, Raymond K. Kostuk

Benjamin D. Chrysler, Yuechen Wu, Zhengshan Yu, Raymond K. Kostuk, "Volume holographic lens spectrum-splitting photovoltaic system for high energy yield with direct and diffuse solar illumination," Proc. SPIE 10368, Next Generation Technologies for Solar Energy Conversion VIII, 103680G (25 August 2017); doi: 10.1117/12.2273204

SPIE Event: SPIE Optical Engineering + Applications, 2017, San Diego, California, United States 


\title{
Volume holographic lens spectrum-splitting photovoltaic system for high energy yield with direct and diffuse solar illumination
}

\author{
Benjamin D. Chrysler ${ }^{*}{ }^{*, b}$, Yuechen $\mathrm{Wu}^{\mathrm{a}}$, Zhengshan $\mathrm{Yu}^{\mathrm{c}}$, Raymond K. Kostuk ${ }^{\mathrm{a}, \mathrm{b}}$ \\ ${ }^{a}$ Electrical and Computer Engineering Dept., University of Arizona, 1230 E Speedway Blvd., \\ Tucson, AZ USA 85721; ${ }^{\mathrm{b}}$ College of Optical Sciences, University of Arizona, 1630 E University \\ Blvd., Tucson, AZ USA 85721; ${ }^{\circ}$ Electrical, Computer, and Energy Engineering, Arizona State \\ University, 650 E Tyler Mall, Tempe, AZ 85287
}

\begin{abstract}
In this paper a prototype spectrum-splitting photovoltaic system based on volume holographic lenses (VHL) is designed, fabricated and tested. In spectrum-splitting systems, incident sunlight is divided in spectral bands for optimal conversion by a set of single-junction PV cells that are laterally separated. The VHL spectrumsplitting system in this paper has a form factor similar to conventional silicon PV modules but with higher efficiencies (>30\%). Unlike many other spectrum-splitting systems that have been proposed in the past, the system in this work converts both direct and diffuse sunlight while using inexpensive 1-axis tracking systems. The VHL system uses holographic lenses that focus light at a transition wavelength to the boundary between two PV cells. Longer wavelength light is dispersed to the narrow bandgap cell and shorter wavelength light to the wide bandgap cell. A prototype system is designed with silicon and GaAs PV cells. The holographic lenses are fabricated in Covestro Bayfol HX photopolymer by 'stitching' together lens segments through sequential masked exposures. The PV cells and holographic lenses were characterized and the data was used in a raytrace simulation and predicts an improvement in total power output of $15.2 \%$ compared to a non-spectrum-splitting reference. A laboratory measurement yielded an improvement in power output of $8.5 \%$.
\end{abstract}

Keywords: spectrum splitting, holographic optical elements, photovoltaics, Covestro Bayfol HX, diffuse sunlight, high efficiency

\section{INTRODUCTION}

According to the Shockley-Quiesser limit the maximum efficiency of a single bandgap PV cell is $33 \%$. In order to exceed this limit several junctions must be used that span the solar spectral range. The most conventional technique to accomplish this is to concentrate sunlight at high levels (>100X) onto multi-junction PV cells that consist of multiple bandgaps deposited in tandem on a common substrate. While the power conversion efficiency of broadband concentrator PV systems is high, they have not gained competitiveness with silicon flat-panel modules due to the complexity and cost of the tracking systems and the inability of high concentration systems to collect diffuse solar power ${ }^{2}$.

An alternative approach to realizing high efficiency solar conversion is to use spectrum-splitting optics in combination with an optimized set of single-junction PV cells that are laterally separated on a receiver plane ${ }^{3,4}$. The spectrum-splitting system (SSS) approach has several advantages over broadband concentrators. Since singlejunction cells are used in parallel the system is not current limited as with tandem multi-junction cells. In addition, since the cells are spatially separated precise lattice matching of different bandgap materials is not required. This leads to lower cell costs. However, in SSSs the complexity is shifted to the optics which must spatially separate different spectral bands in a cost effective and scalable process. Although several SSSs have been demonstrated using dichroic filters ${ }^{5}$ and surface relief type diffractive optical elements ${ }^{6}$, the resulting optical systems have not 
been able to simultaneously allow for large collection apertures, form factors comparable to silicon modules, or the ability to collect both direct and diffuse illumination in a scalable, low cost process.

Optical elements based on volume holography offer a number of advantages for the spectrum-splitting optical problem. In particular, the elements can have close to $100 \%$ diffraction efficiency in a single order, complex functions can readily be recorded, are dispersive, have low scatter, can record high spatial frequencies and corresponding large diffraction angles, and they have been shown to be mass producible in large quantities at low $\operatorname{cost}^{7}$.

In this paper a volume holographic lens (VHL) configuration is used as shown in Fig. 1. The VHL system uses an off-axis holographic lens to focus light at normal incidence in one dimension at a "transition" wavelength to the boundary between narrow bandgap (silicon) and wide bandgap (GaAs) PV cells. Longer wavelength light is dispersed onto silicon and shorter wavelength light is dispersed onto GaAs. Light that is not diffracted is transmitted to the cell underneath the lens. Vorndran et al. ${ }^{8}$ provided a conceptual framework for designing VHL systems and predicted an efficiency of $30.6 \%$ using GaInP and silicon PV cells, however they did not suggest methods for fabricating the system or directly demonstrate its capability.

This paper builds upon the work of Vorndran et al. ${ }^{8}$ with the design of a VHL system that uses GaAs and silicon PV cells, a technique for the fabrication of efficient off-axis holographic lens segments in a single layer of Covestro Bayfol $\mathrm{HX}^{9}$ (formerly Bayer MaterialScience) holographic film, and a laboratory demonstration of the system.

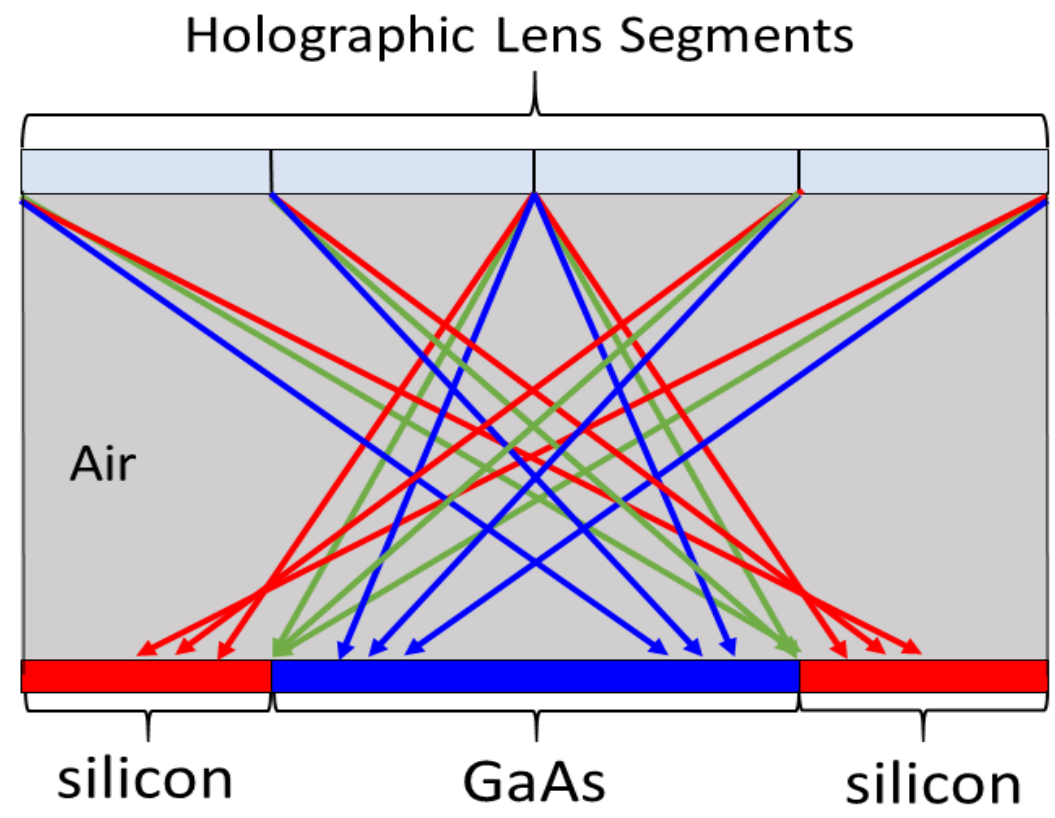

Figure 1: A schematic raytrace of the VHL unit cell used in this work. The unit cell consists of an array of off-axis volume holographic lens segments that focus light at the transition wavelength (the green rays) to the boundary of alternating strips of wide bandgap (GaAs) and narrow bandgap (silicon) PV cells. Longer wavelength light is dispersed onto the silicon cell and shorter wavelength light is dispersed onto the GaAs cell. 


\section{DESIGN AND FABRICATION OF VHL PROTOTYPE SYSTEM}

\subsection{PV Cell Selection}

The VHL prototype system was designed based on the availability of two $16 \mathrm{~mm}$ X $50 \mathrm{~mm}$ PV cells (Table 1): a GaAs cell from Alta Devices and a silicon cell fabricated at Arizona State University. Each PV cell has a spectral response determined in part by its bandgap energy and characterized by its spectral conversion efficiency, $S C E(\lambda)$. $S C E(\lambda)$ is defined as the ratio of electrical power output $P_{\text {elec }}(\lambda)$ over incident optical power $P_{\text {opt }}(\lambda)$ for incident light with wavelength $\lambda$ :

$$
\operatorname{SCE}(\lambda)=\frac{P_{\text {elec }}(\lambda)}{P_{\text {opt }}(\lambda)}
$$

$\operatorname{SCE}(\lambda)$ for each PV cell was measured and plotted in Fig. 2. Spectral bands are defined based on the PV cell that has the highest $\operatorname{SCE}(\lambda)$ for light at wavelength $\lambda$. The spectral band for GaAs is $350 \mathrm{~nm}-875 \mathrm{~nm}$ and for silicon is $875 \mathrm{~nm}-1200 \mathrm{~nm}$. Ideally all incident sunlight within each spectral band will be directed to the corresponding PV cell.

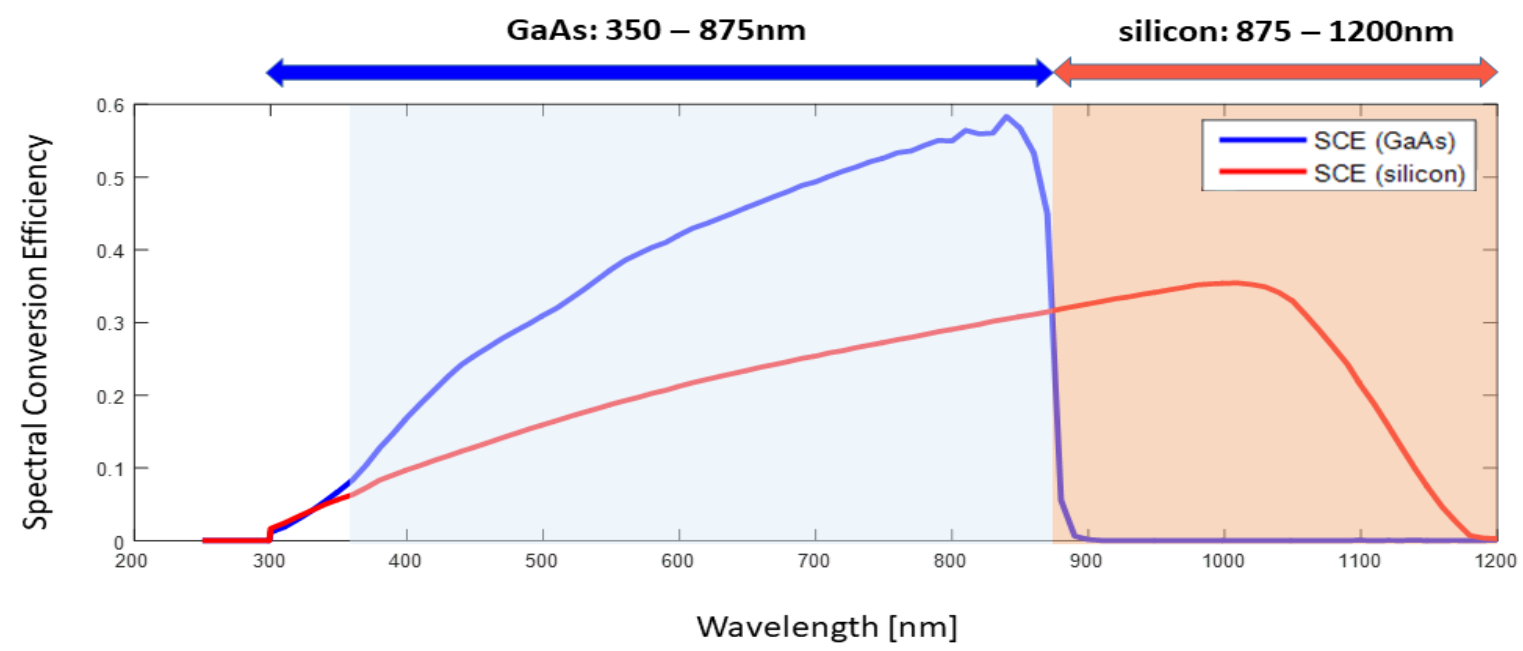

Figure 2: Spectral Conversion efficiency for the GaAs and silicon cells used in the demonstration. Spectral bands are defined by the cell with the highest $S C E(\lambda)$ for light at wavelength $\lambda$. Silicon is more efficient for wavelengths above $875 \mathrm{~nm}$, while GaAs is more efficient below $875 \mathrm{~nm}$.

Table 1: PV cell and system design parameters for VHL prototype

\begin{tabular}{|l|l|l|l|l|r|}
\hline Bandgap & Material & Manufacturer & Width & Length & Efficiency \\
\hline Wide $-1.4 \mathrm{eV}$ & GaAs & Alta Devices & $16 \mathrm{~mm}$ & $50 \mathrm{~mm}$ & $25.6 \%$ \\
\hline Narrow $-1.1 \mathrm{eV}$ & Silicon & Arizona State University & $16 \mathrm{~mm}$ & $50 \mathrm{~mm}$ & $19.6 \%$ \\
\hline
\end{tabular}

\subsection{Design and Fabrication of Holographic Lenses}

The unit cell aperture is divided into four holographic lens segments as depicted in Fig. 3, each with unique diffraction requirements upon reconstruction. The two segments on the left, 'I-L' and 'II-L', focus light at the transition wavelength $\lambda_{T}$ towards the silicon cell on the right. The segments on the right, 'II-R' and 'I-R' focus light at the transition wavelength $\lambda_{T}$ towards the silicon cell on the left. Segments that are positioned over silicon, 'I-L' and 'I-R' are designed to diffract most efficiently in the GaAs spectral band (350-875nm) and segments positioned over GaAs, 'II-L' and 'II-R' are designed to diffract most efficiently in the silicon spectral band (875-1200nm).

These design considerations were characterized by three reconstruction parameters $\lambda_{t}, \theta_{t}$, and $\lambda_{p}$. The transition wavelength $\lambda_{t}$ was determined to be $875 \mathrm{~nm}$ to achieve a sharp spectral cutoff between the PV cells. The angle of 
diffraction $\theta_{t}$ is the diffraction angle at the center of the holographic lens for normally incident light at wavelength $\lambda_{t}$. It was determined based on the geometry of the unit cell: $\left|\theta_{t}\right|=38.7^{\circ}$ for 'I-L' and 'I-R' segments and $\left|\theta_{t}\right|=$ $25.6^{\circ}$ for 'II-L' and 'II-R' segments. The wavelength of peak diffraction efficiency $\lambda_{p}$ was determined based on the spectral band the hologram was designed to diffract to. Due to the limited spectral bandwidth of the hologram, a value was chosen near the center of the corresponding spectral band: $\lambda_{\mathrm{p}}=650 \mathrm{~nm}$ for 'I-L' and 'I-R' segments and $\lambda_{\mathrm{p}}=1000 \mathrm{~nm}$ for 'II-L' and 'II-R' segments.

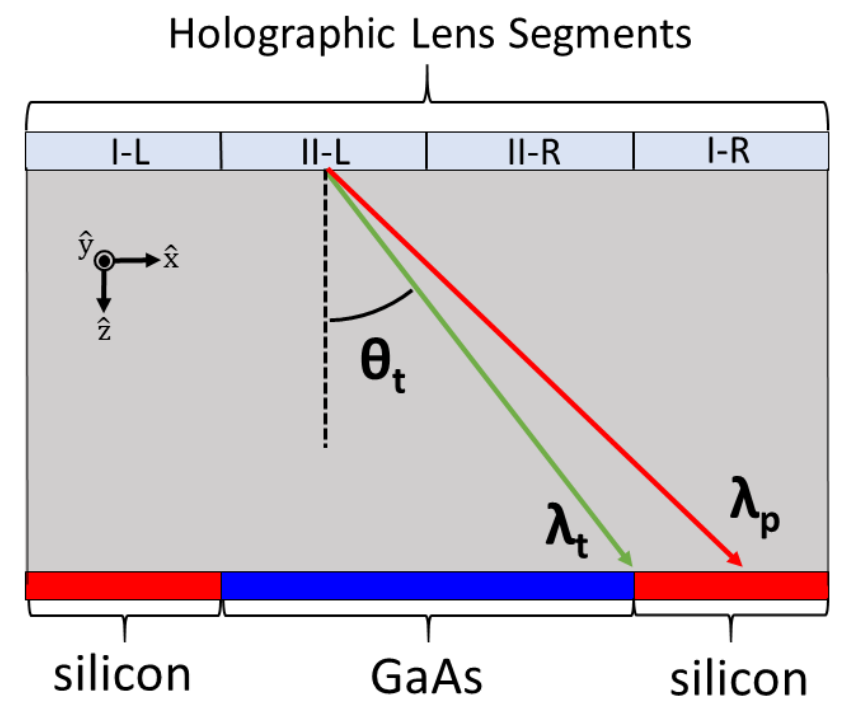

Figure 3: The unit cell aperture is divided into four holographic lens segments 'I-L', 'II-L', 'II-R', and 'I-R'. The reconstruction parameters $\lambda_{t}, \theta_{t}$, and $\lambda_{p}$ are used to calculate the K-vector at the center of each lens segment and in this example are illustrated for segment 'II-L'. Normally incident light at wavelength $\lambda_{t}$ is diffracted from the center of the lens segment at angle $\theta_{t}$ to the intersection of the GaAs and silicon PV cells. Light at wavelength $\lambda_{p}$ is Bragg-matched and has the highest efficiency of the lens segment.

Each holographic lens segment was optimized to meet these considerations. First, the K-vector at the center of the lens segment was determined based on the reconstruction parameters $\lambda_{t}, \theta_{t}, \lambda_{p}$. Next, the construction angles for this $\mathrm{K}$-vector at wavelength $\lambda_{c}$ are calculated. Finally, the positioning of the off-axis cylindrical lens during construction is determined to minimize chromatic aberrations at the transition wavelength.

The two components of the desired K-vector at the center of the lens, $\boldsymbol{K}$, are calculated sequentially. First, $K_{x}$ is calculated based on $\lambda_{t}$ and $\theta_{t}$, while $K_{z}$ remains a free-parameter. Light at wavelength $\lambda_{t}$ has wavevector $\beta_{t}=$ $2 \pi / \lambda_{t}$ and the grating equation for normally incident light yields $K_{x}$ :

$$
\sin \left(\theta_{t}\right)=\frac{K_{x}}{\beta_{t}}
$$

Next, $K_{z}$ is determined based on $\lambda_{p}$ and the calculated value of $K_{x}$. Normally incident light that has wavevector $\beta_{p}=2 \pi n / \lambda_{p}$ is Bragg-matched to maximize diffraction efficiency. $K_{z}$ is calculated through relationships derived from the vector geometry of Fig. 4.a:

$$
K_{z}=\beta_{p}-\sqrt{\beta_{p}{ }^{2}-K_{x}{ }^{2}},
$$

Next, the construction angles for $\boldsymbol{K}$ are calculated for light at the construction wavelength $\lambda_{\mathrm{c}}$ that has wavevector, $\beta_{c}=2 \pi n / \lambda_{c}$. The slant angle, $\phi$, and interbeam angle, $\theta_{i}$, are determined (Fig. 4.b): 


$$
\begin{aligned}
\tan (\phi) & =\frac{K_{z}}{K_{x}}, \\
\sin \left(\frac{\theta_{i}}{2}\right) & =\frac{K / 2}{\beta_{c}},
\end{aligned}
$$

And construction angles within the photopolymer, $\theta_{1}$ and $\theta_{2}$, are calculated:

$$
\begin{aligned}
& \theta_{1}=\phi-\frac{\theta_{i}}{2}, \\
& \theta_{2}=\phi+\frac{\theta_{i}}{2},
\end{aligned}
$$

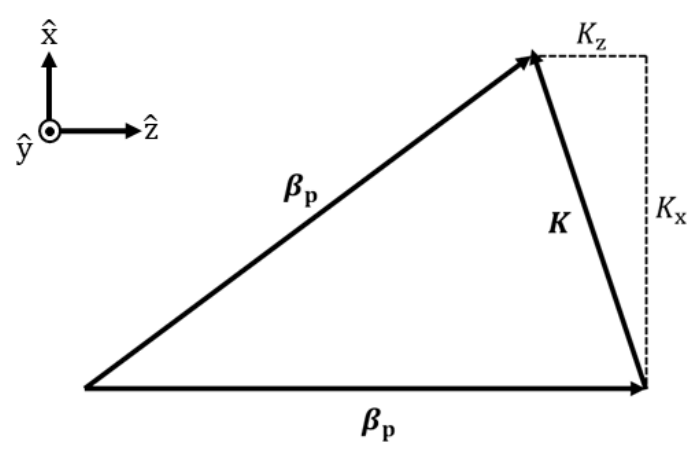

(a)

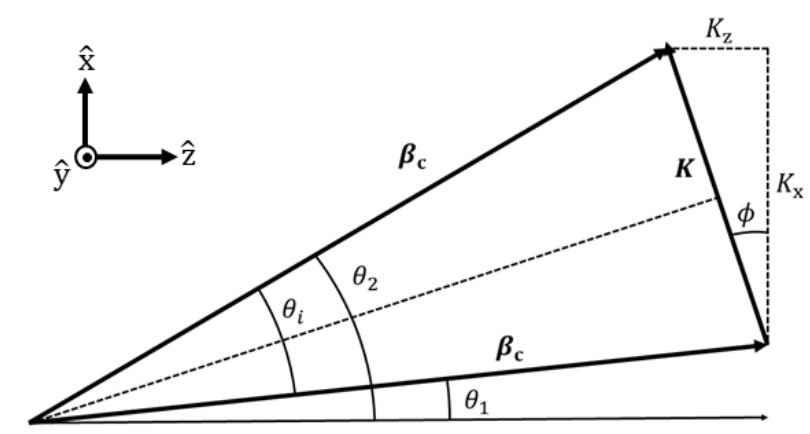

(b)

Figure 4: The reconstruction parameters $\lambda_{t}, \theta_{t}$, and $\lambda_{p}$ are used to determine $\boldsymbol{K} . K_{x}$ is determined from $\lambda_{t}$ and $\theta_{t}$, while $K_{z}$ remains a free-parameter. (a) Normally incident light that has a wavevector $\beta_{p}$ is Bragg matched. The vector geometry is used to derive an equation which determines $K_{z}$. (b) The construction angles $\theta_{1}$ and $\theta_{2}$ for $\boldsymbol{K}$ are determined for light with a wavevector $\beta_{c}$.

The final step in the holographic lens design process is optimizing the lens position ' $\mathrm{d}$ ' in the construction setup depicted in Fig. 5.a. Since the holographic lens is formed at a wavelength $\lambda_{C}$ but focuses at reconstruction wavelength $\lambda_{t}$, raytrace simulations were used to minimize chromatic aberrations. A hologram is formed in a raytrace model in $\mathrm{FRED}^{10}$ using the optical setup in Fig. 5.a. The center of the lens lies along a line at angle $\theta_{2}$ with respect to the center of the hologram being formed. Successive raytrace simulations at the reconstruction wavelength $\lambda_{t}$ are performed while varying lens position ' $\mathrm{d}$ ' in the construction until optimal focusing is achieved.

The holographic lens design parameters and construction setups were determined at a construction wavelength $\lambda_{c}=$ $514 \mathrm{~nm}$ for a separation of $2.5 \mathrm{~cm}$ in air between the photopolymer and the PV cells (table 2). The holographic lenses were fabricated in 16um thick samples of Covestro Bayfol HX (formerly Bayer MaterialScience) photopolymer. The holographic lens segments were integrated in the same optical element by "stitching" lens segments in the photopolymer through sequential masked exposures with a $514 \mathrm{~nm}$ argon laser as depicted in Fig 5.a. A row of four holographic lens segments of dimension $8 \mathrm{~mm}$ X $25 \mathrm{~mm}$ were formed using the construction setups listed in table 2 . A second row used identical construction setups and was required to achieve a $50 \mathrm{~mm}$ aperture due to limitations in the beam expansion optics. 


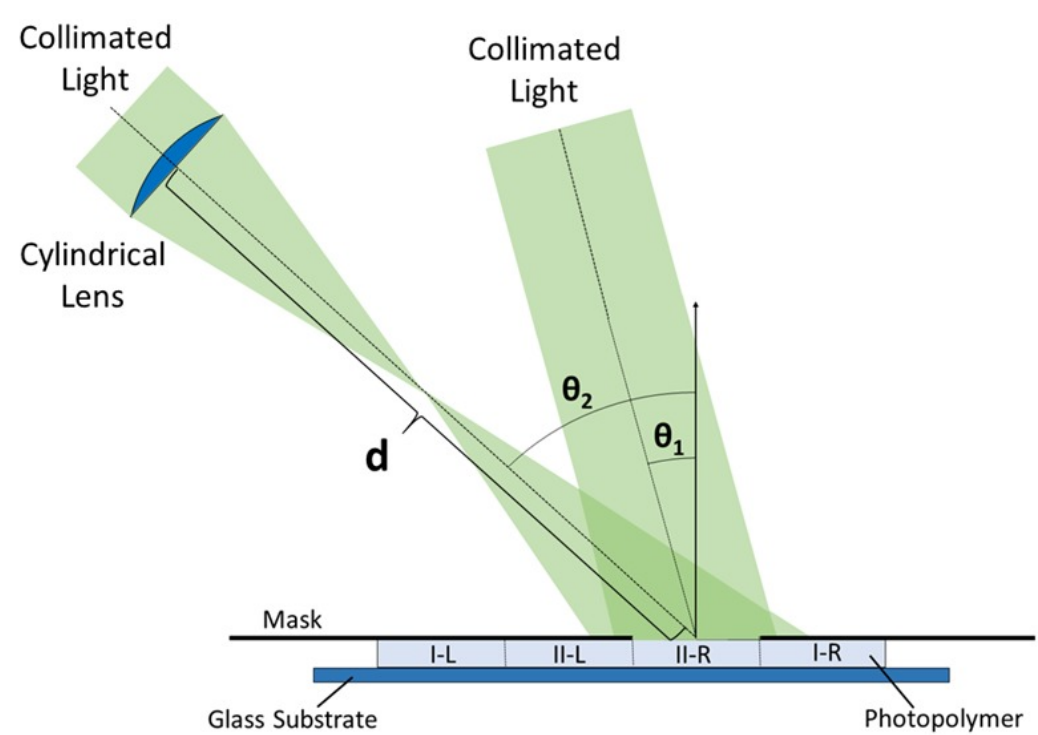

(a)

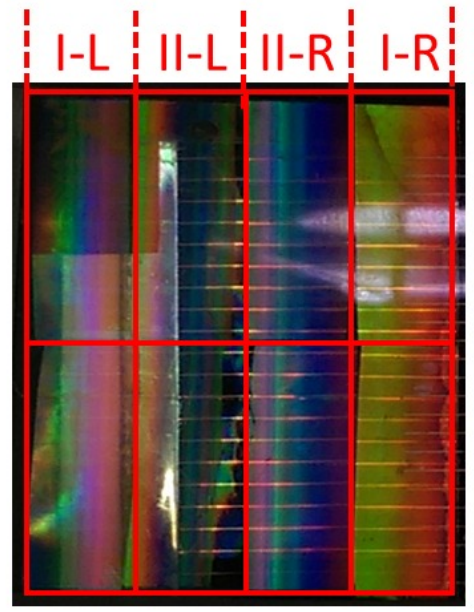

(b)

Figure 5: (a) The cylindrical holographic lens segments were formed with a planar wavefront and a wavefront from an off-axis cylindrical lens with a focal length of $5 \mathrm{~cm}$. Holographic lens segments are constructed sequentially by shifting a mask along the photopolymer surface. (b) The holographic lens array for a unit cell was fabricated in 16um thick Covestro Bayfol HX and consists of eight holographic lens segments with diffraction properties and construction setups listed in table 2.

Table 2: Design parameters for each holographic lens segment and their corresponding construction setups.

\begin{tabular}{|c|c|c|c|c|c|}
\hline & & \multicolumn{4}{|c|}{ Holographic Lens Segment } \\
\hline & & $\mathrm{I}-\mathrm{L}$ & $\mathrm{II}-\mathrm{L}$ & II-R & $\mathrm{I}-\mathrm{R}$ \\
\hline \multirow{3}{*}{ Design } & $\lambda \mathrm{t}[\mathrm{nm}]$ & 875 & 875 & 875 & 875 \\
\hline & $\theta t[\mathrm{deg}]$ & 38.7 & 25.6 & -25.6 & -38.7 \\
\hline & $\lambda \mathrm{p}[\mathrm{nm}]$ & 650 & 1000 & 1000 & 650 \\
\hline \multirow{3}{*}{ Construction } & $\theta 1$ (in air) [deg] & -2.8 & -7.0 & 7.0 & 2.8 \\
\hline & $\theta 2$ (in air) [deg] & -24.6 & -22.1 & 22.1 & 24.6 \\
\hline & $\mathrm{d}[\mathrm{mm}]$ & 125 & 100 & 100 & 125 \\
\hline
\end{tabular}

\section{VHL PROTOTYPE SYSTEM EVALUATION}

\subsection{Performance Metrics and Demonstration Layout}

Performance of the VHL system is evaluated by measuring Power Boost (PB), or the ratio of the combined power output of the PV cells $P_{G a A s, V H L}+P_{S i, V H L}$ with holographic lenses for spectrum splitting (Fig. 6.a), compared to the combined power output of the PV cells in a reference measurement $P_{G a A s, r e f}+P_{\text {Siref }}$, without holographic lenses and no spectrum splitting (Fig 6.b) 


$$
P B=\frac{P_{G a A s, V H L}+P_{S i, V H L}}{P_{G a A s, r e f}+P_{\text {Siref }}}
$$

The layout of the reference measurement is similar to that of the VHL, but the holographic lenses are replaced with an unexposed piece of photopolymer that was cured with a UV lamp and does not diffract light. This was chosen to account for Fresnel reflections and absorption in the photopolymer material during the measurement.

The holographic lens arrays for two unit cells were fabricated and placed adjacent to each other as depicted in Fig. 6.a.. The silicon cell is placed in the center and spans across both unit cells, and the GaAs cell is placed under the unit cell to the left. This configuration was chosen to accommodate the size of the silicon cell and reduce the number of measurements required. The combined power generated by the two PV cells is equivalent to the power output of a unit cell.

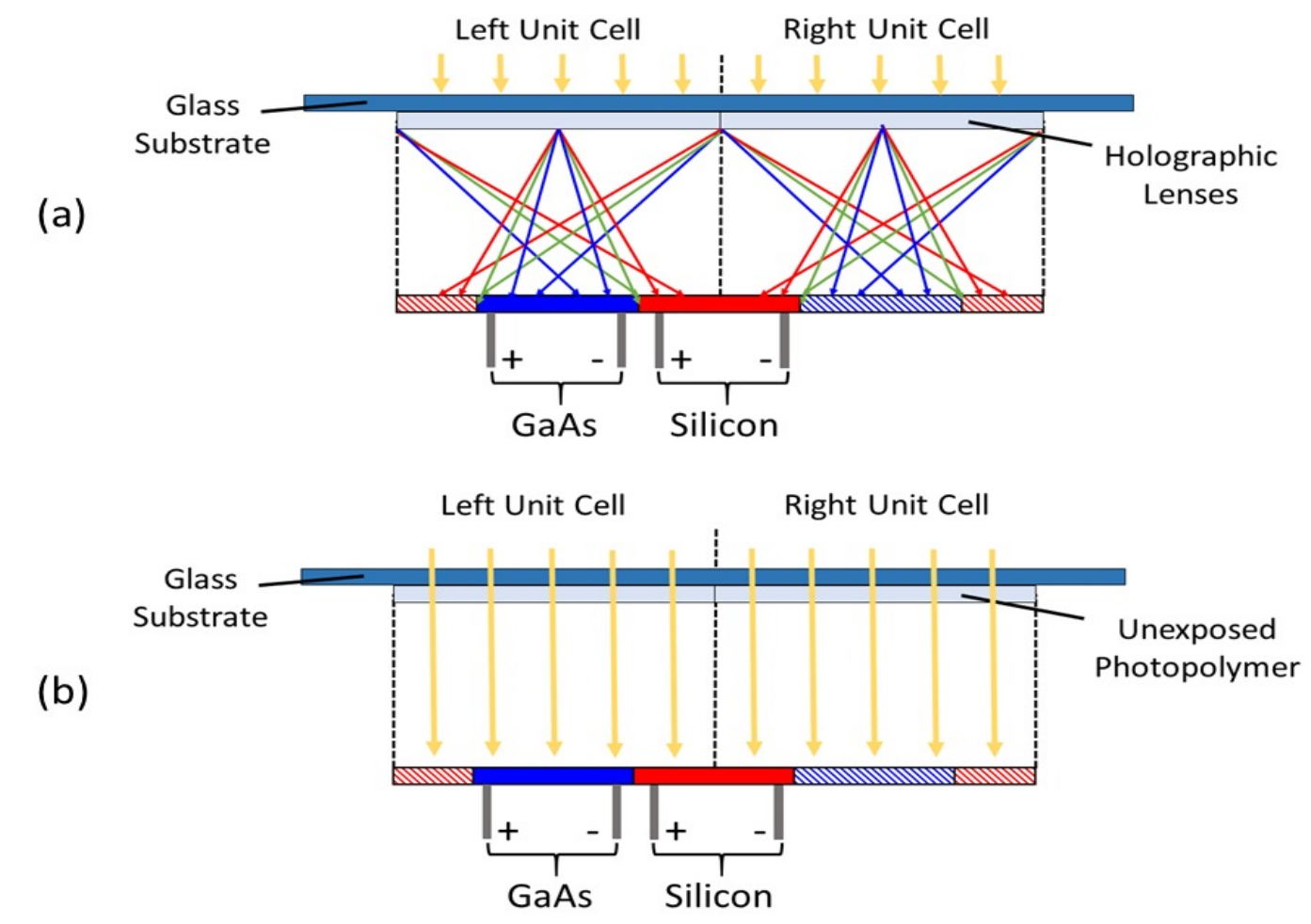

Figure 6: (a) Measurement of the power output of GaAs and silicon PV cells using holographic lenses for spectrum splitting. (b) Measurement of reference power output using glass/photopolymer interface without holographic lenses.

\subsection{Predicted System Performance}

The optical performance of the system was evaluated through the Spectral Optical Efficiency, $\operatorname{SOE}(\lambda)$, for each PV cell (Fig. 7). $\operatorname{SOE}(\lambda)$ is the ratio of optical power at wavelength $\lambda$ incident upon the PV cell $P_{\text {cell }}(\lambda)$ over the optical power incident upon the unit cell aperture $P_{\text {inc }}(\lambda)$

$$
\operatorname{SOE}(\lambda)=\frac{P_{\text {cell }}(\lambda)}{P_{\text {inc }}(\lambda)}
$$

$\operatorname{SOE}(\lambda)$ can be determined by measuring the diffraction efficiency of each holographic lens segment and incorporating that data in a raytrace simulation. The diffraction efficiency of each lens was determined through the spectral transmittance. Light that was not transmitted was assumed to be diffracted and the measurements were normalized to account for Fresnel reflections and absorption. The measured diffraction efficiency values were 
assigned to holographic lens elements in a FRED raytrace model. A uniformly weighted broadband source with power $P_{\text {inc }}(\lambda)=1$ was used so that the power read by a detector placed at the PV cell, $P_{\text {cell }}$, directly yielded $\operatorname{SOE}(\lambda)$ in accordance with equation 8 . The measured optical performance $\operatorname{SOE}(\lambda)$ and cell performance $\operatorname{SCE}(\lambda)$ were used along with the $A M 1.5(\lambda)$ solar spectrum to determine cell power output.

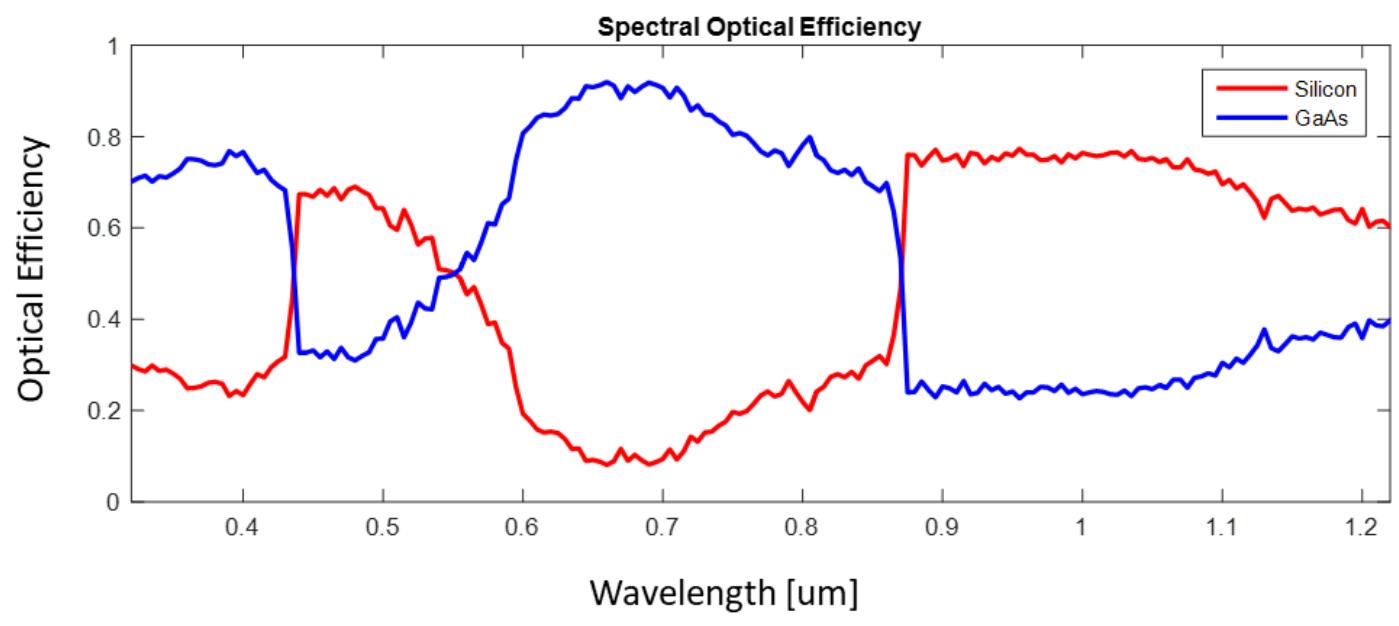

Figure 7: Spectral Optical Efficiency for silicon and GaAs obtained by incorporating measured diffraction efficiencies of the holographic lenses into a raytrace model in FRED.

The power generated by the GaAs cell is given by:

$$
P_{G a A s, V H L}=\int A M 1.5(\lambda) \cdot S O E_{G a A s}(\lambda) \cdot S C E_{G a A s}(\lambda) \cdot d \lambda,
$$

The power generated by the silicon cell is given by:

$$
P_{S i, V H L}=\int A M 1.5(\lambda) \cdot S O E_{S i}(\lambda) \cdot S C E_{S i}(\lambda) \cdot d \lambda,
$$

The power of the reference measurements can be determined based on the cell efficiencies listed in table 1 and the total incident power in the $A M 1.5(\lambda)$ spectrum, $P_{A M 1.5}$ :

$$
\begin{gathered}
P_{\text {GaAs,ref }}=\frac{1}{2} P_{A M 1.5} \cdot \eta_{\text {GaAs }}, \\
P_{\text {Siref }}=\frac{1}{2} P_{A M 1.5} \cdot \eta_{S i},
\end{gathered}
$$

Where the factor $1 / 2$ is used because each cell has equal dimensions and receives $1 / 2$ of the total incident power.

Using equations 9-12 to determine the power output of each PV cell, the predicted power boost is determined: $\mathrm{PB}_{\text {predicted }}=15.2 \%$.

\subsection{Measured System Performance}

The prototype VHL module was illuminated with a Xenon arc lamp solar simulator (Fig 8.a) in the configuration of Fig 6.a. The IV-curves of both PV cells were measured with a Keithley 2440 sourcemeter (Fig. 8.b,c). The power output of the PV cells was determined using the maximum power points of the IV-curves. The reference power output was measured in a similar manner after replacing the holographic lenses with unexposed photopolymer in the configuration of Fig 6.b. 
There is a reduction in power output of the Silicon cell (-26.5\%), and an increase in the power output of the GaAs cell $(+36.9 \%)$ resulting in a net increase in total power output of $\mathrm{PB}_{\text {meas }}=+8.5 \%$. The decrease in power output of the silicon cell is expected and is the result of the relatively narrow spectral band that is directed to silicon $(875 \mathrm{~nm}-$ $1200 \mathrm{~nm})$ vs. $(300 \mathrm{~nm}-875 \mathrm{~nm})$ directed to GaAs and because light within the GaAs spectral band is capable of being converted by silicon, but light within the silicon spectral band is not capable of being converted by GaAs.

(a)

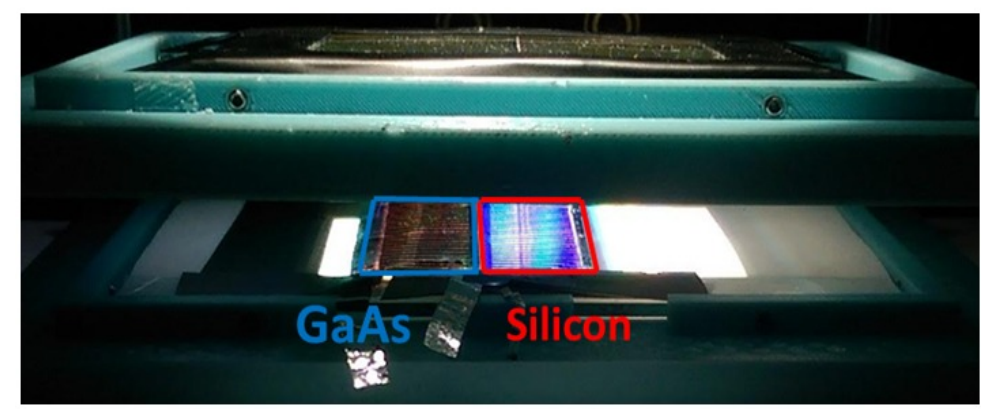

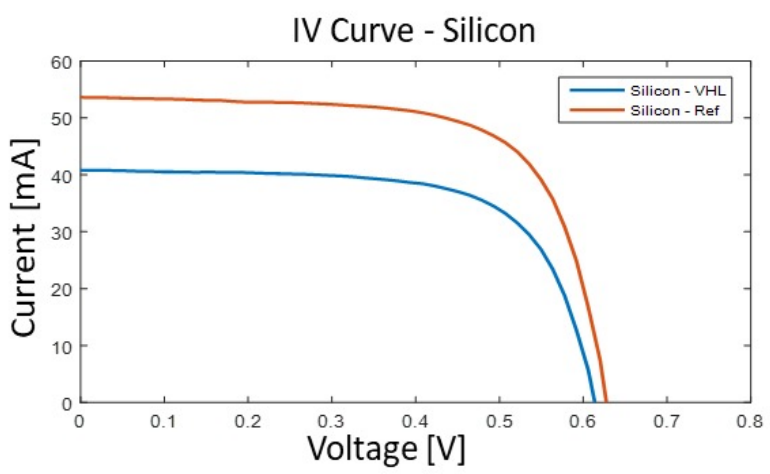

(b)

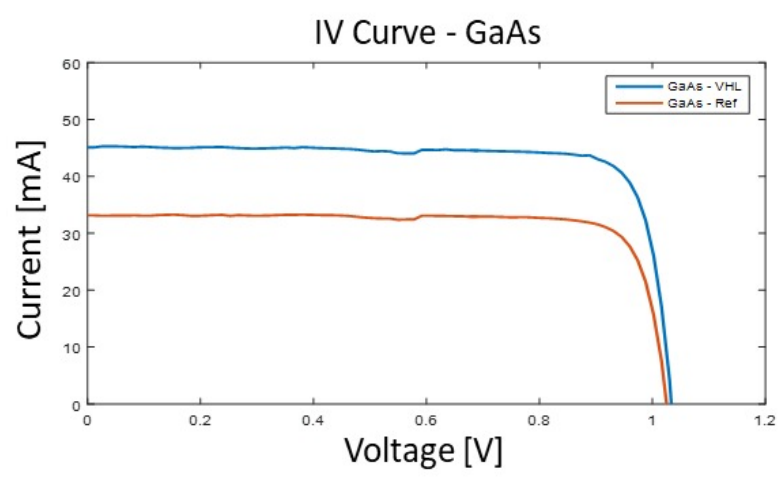

(c)

Figure 8: (a) The holographic lens elements were placed in a holder and illuminated with a Xenon arc lamp solar simulator as depicted in Fig 6.a. IV-curve for silicon (b) and GaAs (c) are shown with and without the holographic lenses. The blue curves are with the holographic lenses, and the orange curves are the reference.

\section{CONCLUSION}

A prototype Volume Holographic Lens (VHL) spectrum splitting photovoltaic system was designed, fabricated, and tested. Holographic lenses were designed to separate incident sunlight into two spectral bands tailored for efficient conversion by silicon and GaAs PV cells. The holographic lens segments were optimized for construction with collimated light and a wavefront from an off-axis cylindrical lens. Several holographic lens segments were "stitched" together on a single piece of Covestro Bayfol HX photopolymer. The performance of the PV cells and holographic lenses were evaluated and incorporated in a raytrace model to calculate the predicted Power Boost of the prototype system. The experimental results were obtained by illuminating the system with a solar simulator and measuring the power output of each PV cell for spectrum-splitting and reference configurations. The measured increase in power output of the VHL prototype system (8.5\%) shows general agreement with the predicted result $(15.2 \%)$.

\section{ACKNOWLEDGEMENTS}

The authors wish to acknowledge support from the NSF/DOE ERC cooperative agreement No. EEC-1041895 and NSF grant ECCS-1405619. Ben Chrysler would also like to acknowledge partial support from the State of Arizona 
TRIF/WEES Grant 5835204 and the National Science Foundation Graduate Research Fellowship Program Grant (DGE-1143953). Any opinions, findings, and conclusions or recommendations expressed in this material are those of the authors and do not necessarily reflect the views of the National Science Foundation.

\section{REFERENCES}

[1] Shockley, W., Queisser, H. J., "Detailed balance limit of efficiency of p n junction solar cells," Journal of applied physics, 32(3), 510-519 (1961).

[2] Philipps, S. P., Andreas W. B., Horowitz K., and Kurtz S., "Current status of concentrator photovoltaic (CPV) technology," NREL, < http://www.nrel.gov/docs/fy16osti/65130.pdf > (2015).

[3]Victoria C. M., Chemisana D., and Atencia J., "Holographic solar energy systems: The role of optical elements," Renewable and Sustainable Energy Reviews 59, 130-140 (2016).

[4] Russo, J. M., Zhang, D., Gordon, M., Vorndran, S., Wu, Y., \& Kostuk, R. K, "Spectrum splitting metrics and effect of filter characteristics on photovoltaic system performance," Optics express, 22(102) A528-A541 (2014).

[5] Allen B., Kirkpatrick D., Honsberg C., Moore D., Wanlass M., Emery K., Schwartz R., Carlson D., Bowden S., Aiken D., Gray, A., Kurtz, S., Kazmerski L., Steiner, M., Gray, J., Davenport, T., Buelow R., Takacs, L., Shatz, N., Bortz, J., Jani, O., Goosen, K., Kiamilev, F., Doolittle, A., Fergson, I., Unger, B., Shmidt, G., Christensen, E., Salzman, D., "Very high efficiency solar cell modules," Progress in Photovoltaics: Research and Applications 17(1), 75-83 (2009).

[6] Kim, G., Dominguez-Caballero, J. A., Lee, H., Friedman, D. J., \& Menon, R, "Increased photovoltaic power output via diffractive spectrum separation," Physical review letters 110(12) (2013).

[7] Aspnes, E. D., Castillo, J. E., Courreges, R. D., Hauser, P. S., Rosenberg, G., \& Russo, J. M, "Volume hologram replicator for transmission type gratings" U.S. Patent and Trademark Office. U.S. Patent No. 8,614,842 (2013).

[8] Vorndran, S. D., Chrysler, B., Wheelwright, B., Angel, R., Holman, Z., \& Kostuk, R, "Off-axis holographic lens spectrum-splitting photovoltaic system for direct and diffuse solar energy conversion," Applied optics 55(27) (2016).

[9] Julia M., Atencia J., Chemisana D., and Collados M., "Characterization of volume holographic optical elements recorded in Bayfol HX photopolymer for solar photovoltaic applications," Optics express 24(6), A720-A730 (2016).

[10] "What is FRED," Photon Engineering, < http://photonengr.com/fred-software/> (2017). 